13

\title{
Оптимизация многоострийных полевых эмиттеров с двуслойными защитными покрытиями
}

\author{
(ㄷ Г.Г. Соминский, Е.П. Тарадаев
}

Санкт-Петербургский политехнический университет Петра Великого, 195251 Санкт-Петербург, Россия

e-mail: sominski@rphf.spbstu.ru

(Поступило в Редакцию 22 июня2016 г.)

\begin{abstract}
Проведены расчеты, необходимые для оптимизации упорядоченных многоострийных кремниевых полевых эмиттеров с двуслойными защитными металл-фуллереновыми покрытиями, предназначенных для использования в высоковольтных электронных приборах, работающих в техническом вакууме. Расчеты выполнены с использованием программы COMSOL. Определено влияние на эмиссионные характеристики эмиттеров морфологии их поверхности, в том числе высоты острий и радиуса их вершины, а также расстояния между остриями. Показано, что эмиттеры площадью $0.2 \mathrm{~cm}^{2}$, работающие в условиях частичной взаимной экранировки, могут обеспечить при достаточно больших напряжениях токи порядка нескольких сотен миллиампер.
\end{abstract}

DOI: 10.21883/JTF.2017.06.44519.1949

\section{Введение}

Холодные полевые эмиттеры привлекательны для использования в вакуумной электронике прежде всего потому, что не требуют накала и практически безынерционны. Наиболее простые по конструкции одноострийные полевые эмиттеры традиционно и успешно используются при создании, например, электронных микроскопов и сенсоров, где необходимы, как правило, токи с эмиттера не более $10^{-7}-10^{-5} \mathrm{~A}$.

В последние годы делаются попытки освоить не совсем обычную область применения полевых эмиттеров, использовать их при создании некоторых типов высоковольтных, но миниатюрных электронных приборов, таких, например, как СВЧ усилители и генераторы миллиметрового и субмиллиметрового диапазонов (см., например, $[1,2])$, а также портативных источников рентгеновского излучения (например, $[3,4])$. В указанных приложениях полевые эмиттеры должны обеспечивать одновременно не только высокие плотности тока эмиссии (порядка или более $100-150 \mathrm{~mA} / \mathrm{cm}^{2}$ ), но и большие полные токи (свыше нескольких десятков миллиампер), причем в условиях технического вакуума $[5,6]$.

Применению полевых эмиттеров в высоковольтных приборах в условиях технического вакуума препятствует невысокая их долговечность. Основные причины выхода из строя полевых эмиттеров в высоковольтных устройствах - распыление их поверхности под действием бомбардировки ионами остаточного газа, а также их разрушение из-за перегрева эмитирующих электроны структур при отборе больших токов и/или под действием пондеромоторных сил. Кардинально уменьшить влияние на работу эмиттеров тепловых эффектов и действия пондеромоторных сил можно, используя распределеннные многоострийные катоды, при эксплуатации которых с каждого острия отбираются достаточно малые токи $\leq 3-5 \mu \mathrm{A}$ [6]. Однако и в этом случае необходимо свести к допустимому минимуму разрушающее действие ионной бомбардировки.

Перспективный способ борьбы с действием ионной бомбардировки был выявлен в работах [7-11], где авторы использовали для решения этой проблемы защитные фуллереновые покрытия, активированные потоком ионов калия.

В настоящее время наиболее хорошо отработана и достаточно проста технология создания многоострийных кремниевых структур [12]. По этой причине они представляются перспективными для создания полевых эмиттеров. Но применение таких структур для формирования электронных потоков большой интенсивности затруднено, причем не только потому, что они разрушаются под действием ионной бомбардировки, но и по той причине, что кремниевые острия имеют зачастую малую проводимость. Кроме того, острийные кремниевые эмиттеры бывают недостаточно прочны и часто разрушаются под действием пондеромоторных сил уже при умеренных значениях электрического поля. Как было показано в работах $[6,11,13]$, указанные выше проблемы могут быть решены, если создать на поверхности кремниевого эмиттера специальное двуслойное металлфуллереновое покрытие. Для повышения проводимости и прочности многоострийных эмиттеров на их поверхность напылялось молибденовое покрытие толщиной 5-15 nm. Для защиты от разрушающего действия ионной бомбардировки поверх слоя металла наносилось, а затем активировалось потоком медленных (с энергией $\sim 40 \mathrm{eV}$ ) ионов калия покрытие из молекул фуллерена $\mathrm{C}_{60}$. В результате ионной обработки работа выхода $е \varphi$ эмиттера с двуслойным покрытием понижалась от $5.3 \mathrm{eV}$ (значения, характерного для чисто фуллеренового покрытия) приблизительно до $4.0 \mathrm{eV}$ из-за формирования в покрытии металлофуллеренов типа эндо- $\left(\mathrm{C}_{60} @ \mathrm{~K}\right)$ и/или экзоэдралов (K@ $\left.\mathrm{C}_{60}\right)$. 
Проведенные авторами исследования свидетельствуют, что многоострийные кремниевые эмиттеры площадью $0.2-0.3 \mathrm{~cm}^{2}$ с активированными двуслойными металл-фуллереновыми покрытиями достаточно стабильно работают при токах эмиссии ориентировочно до $50 \mathrm{~mA}$ и при плотностях тока свыше $100 \mathrm{~mA} / \mathrm{cm}^{2}$. Однако, с нашей точки зрения, существуют возможности заметного улучшения эмиссионных характеристик таких катодов, если оптимизировать морфологию их поверхности.

Ранее уже делались попытки оптимизировать морфологию поверхности многоострийных полевых эмиттеров [14,15]. Основное внимание при этом уделялось определению условий, в которых отсутствует или снижена взаимная экранировка острий на поверхности многоострийной системы. Благодаря этому удавалось уменьшить рабочие напряжения, необходимые для получения фиксированных значений электрического поля у поверхности острий. Однако при эксплуатации полевых эмиттеров в высоковольтных электронных прибоpax зачастую важнее обеспечить получение большего тока эмиссии с ограниченной по размерам площади эмиттера при фиксированном и достаточно большом напряжении. В настоящей работе выполнены расчеты, позволяющие выбрать морфологию поверхности многоострийного эмиттера, необходимую для решения именно этой задачи.

\section{Методика расчетов}

Были рассчитаны эмиссионные характеристики острийных катодов с активированными металл-фуллереновыми покрытиями, в том числе уединенного острия, a также многоострийных структур с фиксированной площадью $S=0.2 \mathrm{~cm}^{2}$ эмитирующей поверхности. Варьировали в широких пределах высота острий $h(10 \leq$ $\leq h \leq 60 \mu \mathrm{m})$ и радиус $R$ их вершины $(15 \leq R \leq 40 \mathrm{~nm})$. В многоострийных структурах варьировали, кроме того, количество острий $N(1 \leq N \leq 10000)$ на эмитирующей поверхности и расстояние $L$ между ними $(0.1 \leq$ $\leq L / h \leq 10)$. Диаметр основания острий в расчетах не менялся и был равен $5 \mu \mathrm{m}$.

Расчеты выполнены для диодной системы с бесконечно протяженными плоскими катодом и анодом, расположенными на расстоянии $1.5 \mathrm{~mm}$ друг от друга. Форма острий задавалась в виде, показанном на рис. 1. Именно такую форму имеют острия, изготавливаемые с использованием хорошо отработанных к настоящему времени технологий [12]. Для описания распределений электрического поля и плотностей тока электронов с поверхности острия удобно ввести криволинейную координату $l$. Начало $l=0$ и направление отсчета координаты $l$ указаны на рис. 1 .

Работа выхода острий была принята в расчетах равной $4.0 \mathrm{eV}$, т.е. типичной для эмиттеров с активированными металл-фуллереновыми покрытиями. Напряжение $U$

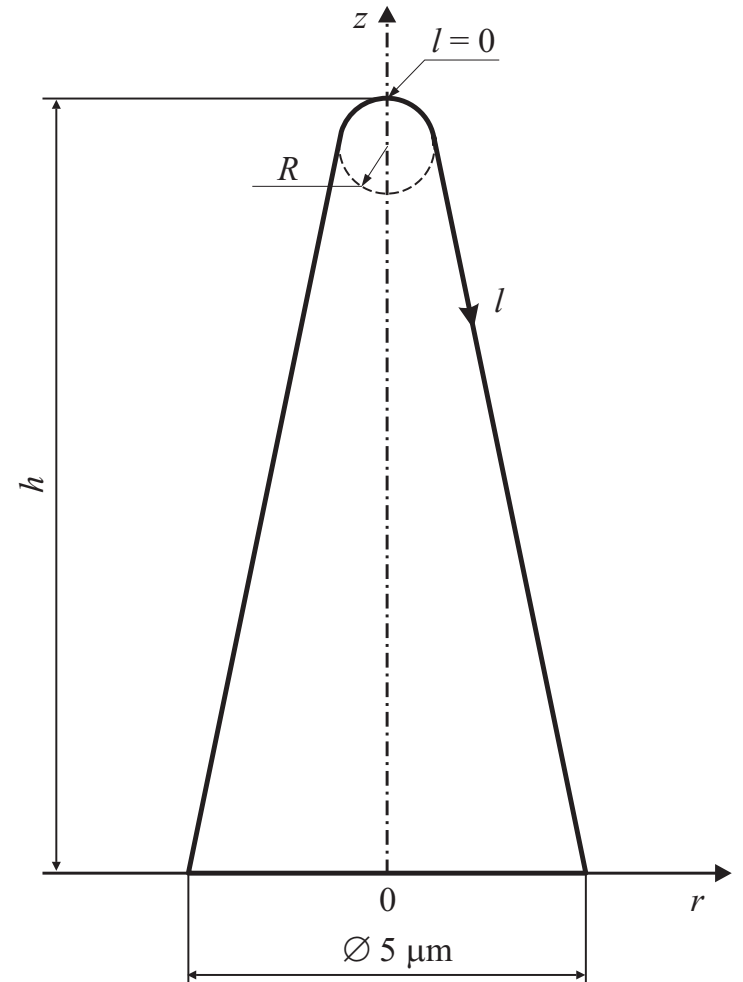

Рис. 1. Схематическое изображение сечения острия. $h$ и $R$ - соответственно высота острия и радиус закругления его вершины.

между катодом и анодом диодной структуры менялось в пределах $1 \leq U \leq 20 \mathrm{kV}$.

С использованием программы COMSOL определялось распределение электрического поля $E$ у поверхности острий, а затем с помощью формулы Фаулера-Нордгейма рассчитывались распределение плотности тока полевой эмиссии $j(l)$ с их поверхности и полный ток с каждого острия. Для определения полного тока полевой эмиссии многоострийного катода суммировались токи со всех острий.

\section{Результаты расчетов}

Одним из основных параметров, определяющих эмиссионные свойства острийных катодов, является коэффициент усиления поля $\beta=E / \bar{E}$, равный отношению максимальной напряженности электрического поля $E_{\max }$ у вершины острия к среднему значению поля, определяемому отношением $\bar{E}=U / d$, где $d-$ расстояние между катодом и анодом.

На рис. 2 показаны типичные рассчитанные распределения электрического поля $E(l)$ и плотности тока эмиссии $j(l)$ для уединенного острия при фиксированных $h=30 \mu \mathrm{m}, R=20 \mathrm{~nm}$ и анодном напряжении $U=9 \mathrm{kV}$. С ростом $l$ (при удалении от вершины острия) происходит быстрое падение электрического поля и плотности тока. Так, например, значение $j$ уменьшается 
на несколько порядков уже на расстоянии $l \sim 1.5 R$ от вершины. В приведенном примере полный ток со всего острия был приблизительно равен $1 \mu \mathrm{A}$ при максимальной плотности тока $\sim 1.5 \cdot 10^{5} \mathrm{~A} / \mathrm{cm}^{2}$.

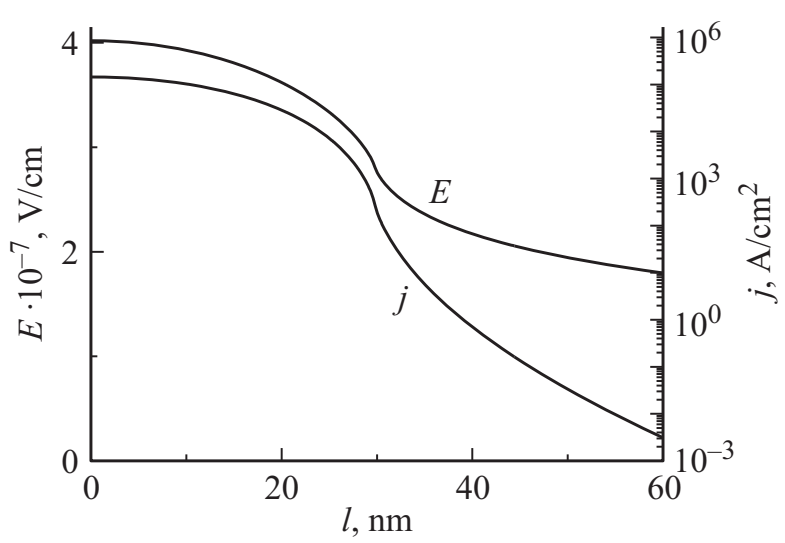

Рис. 2. Типичные распределения электрического поля $E$ и плотности тока эмиссии $j$ для уединенного острия при напряжении между катодом и анодом $U=9 \mathrm{kV}$. Расчет проведен для $h=30 \mu \mathrm{m}, R=20 \mathrm{~nm}, e \varphi=4.0 \mathrm{eV}$.
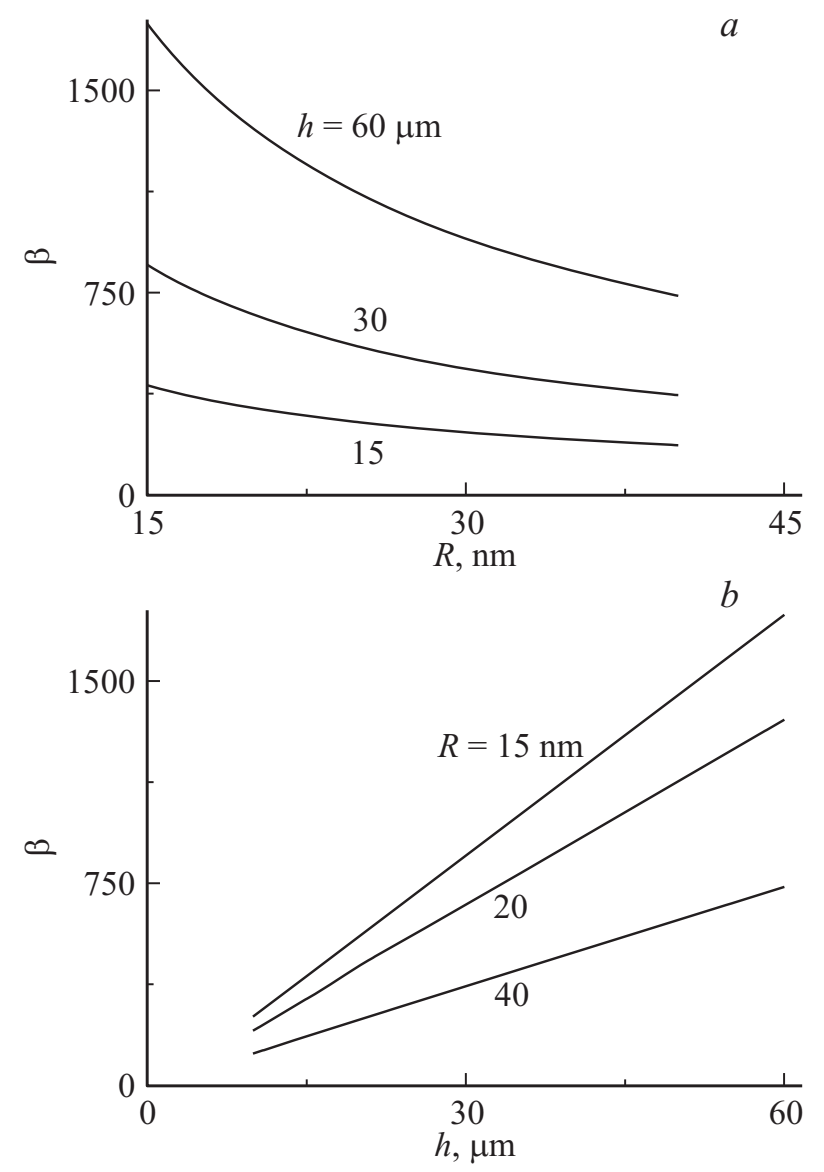

Рис. 3. Зависимости коэффициента усиления поля $\beta$ уединенного острия: $a$ - от радиуса $R$ закругления его вершины при фиксированных значениях высоты острия $h ; b-$ от высоты острия при фиксированном значении радиуса $R$ закругления его вершины.
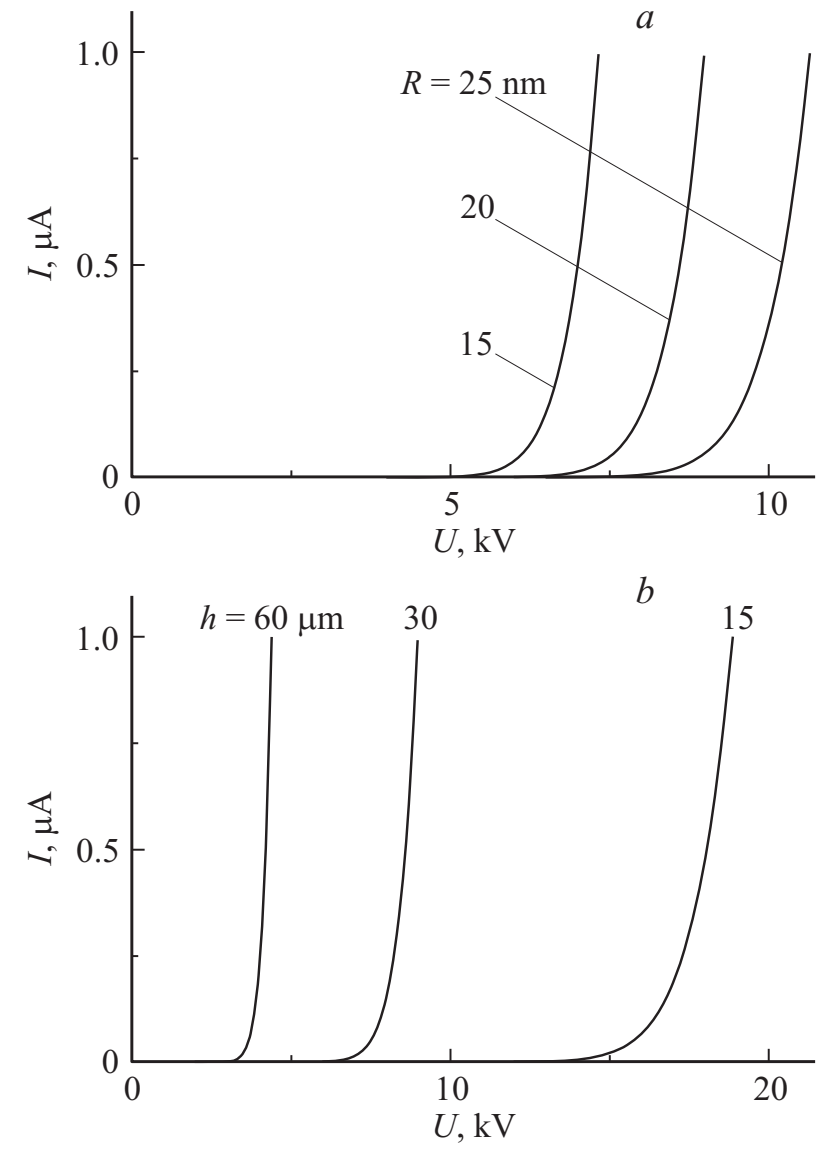

Рис. 4. Вольт-амперные характеристики уединенного острия высотой $h=30 \mu \mathrm{m}$ при различных значениях радиуса его вершины $(a)$. Вольт-амперные характеристики уединенного острия с радиусом вершины $R=20 \mathrm{~nm}$ при различных значениях его высоты $(b)$.

Коэффициент $\beta$ усиления поля одиночного острия определяется его аспектным отношением, величиной отношения $h / 2 R$. При фиксированной высоте острий коэффициент усиления $\beta$ растет с уменьшением их радиуса, а при фиксированном радиусе увеличивается с ростом высоты острий (рис. 3). Изменение $\beta$ сопровождается изменением отбираемых токов, что иллюстрирует рис. 4 . С ростом $\beta$ вольт-амперные характеристики смещаются в область меньших напряжений.

Приведенные данные для одиночных кремниевых острий конической формы хорошо согласуются с полученными ранее результатами расчетов $[14,15]$, выполненных для эмиттеров на основе цилиндрических углеродных нанотрубок. Это не вызывает удивления, так как судя по приведенным выше данным эмиссия острийных катодов определяется в основном торцевой поверхностью катода (рис. 2) и слабо зависит от формы его основания.

В структуре с количеством острий $N>1$ коэффициент усиления поля меняется не только с изменением аспектного отношения острий $h / 2 R$. Его величина уменьшается по сравнению с максимальным значением, 
характерным для одиночного острия, из-за эффекта взаимной экранировки острий $[14,15]$. При увеличении количества острий в структуре это происходит из-за уменьшения расстояния между ними.

В расчетах были получены зависимости коэффициента усиления электрического поля у вершины острий многоострийной структуры от их высоты и расстояния между ними. На рис. 5. показана зависимость коэффициента усиления $\beta$, отнесенного к его максимальному значению $\beta_{\max }$, характерному для уединенного острия, от величины отношения расстояния $L$ между остриями к их высоте $h$. На рис. 5 приведена типичная зависимость, рассчитанная для острий высотой $30 \mu \mathrm{m}$ с радиусом вершины $20 \mathrm{~nm}$.

Из расчета следует, что при расстоянии между остриями $L \leq 2 h$ экранировка острий мала и приближается к коэффициенту усиления уединенного острия. При $L<2 h$ величина $\beta / \beta_{\max }$ падает с уменьшением отношения $L / h$. В связи с этим для получения фиксированного тока при $L<2 h$ требуются большие напряжения. Однако, как уже упоминалось во Введении, для получения токов эмиссии, достаточных для функционирования высоковольтных электронных приборов, допустима работа катода в условиях частичной экранировки. Учитывая тот факт, что острия существующих кремниевых катодов выдерживают отбор токов $I$, не превышающих некоторое предельное значение $I_{\text {extrem }} \sim 3-5 \mu \mathrm{A}$, и разрушаются при больших токах, при выборе оптимальной морфологии поверхности многоострийных катодов для высоковольтных приборов приходится принимать компромиссное решение, при котором, с одной стороны, на поверхности катода должно быть создано достаточно большое количество острий $N$, удовлетворяющих соотношению

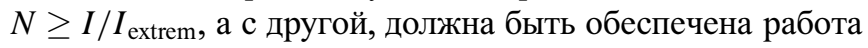
электронного прибора при повышенном рабочем напряжении.

Для принятия такого компромиссного решения необходимы данные о влиянии морфологии поверхности многоострийных катодов на отбор тока с их поверхности. На рис. 6 приведено семейство вольт-амперных харак-

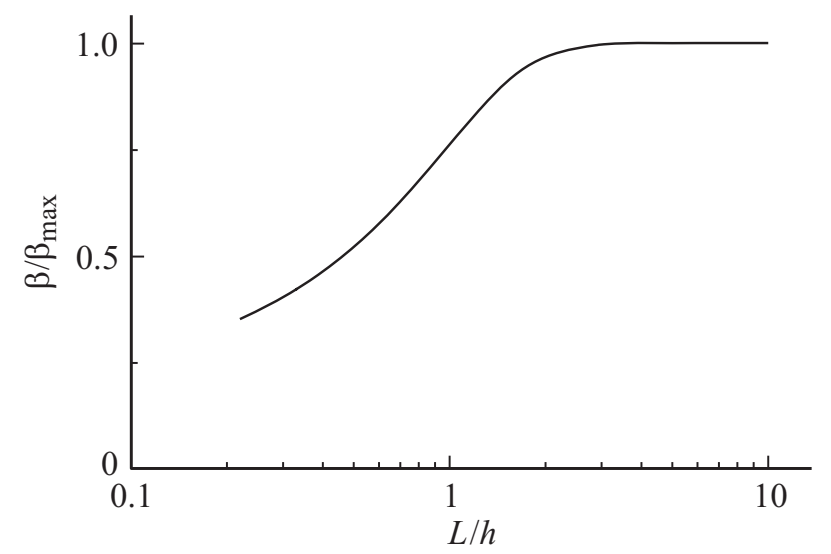

Рис. 5. Типичная зависимость коэффициента усиления $\beta$ одиночного острия в многоострийном катоде от отношения расстояния между остриями к их высоте.

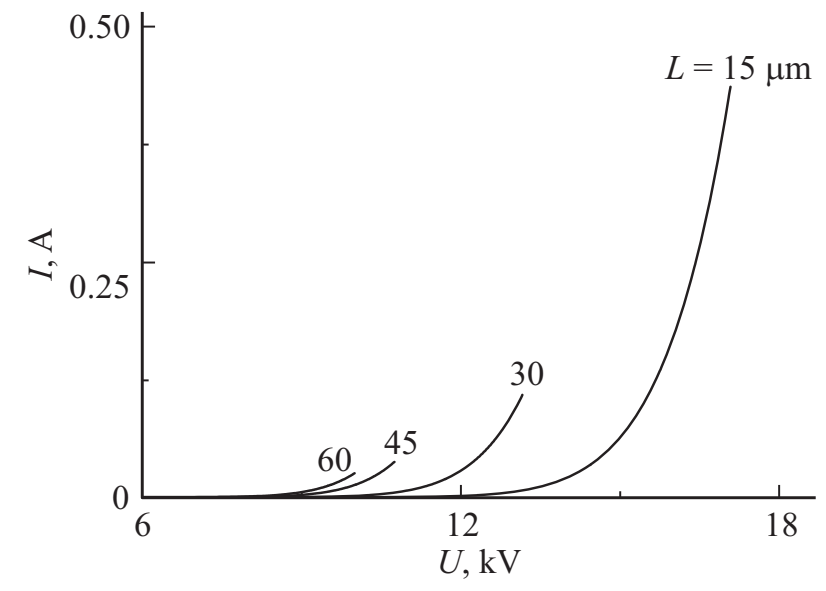

Рис. 6. Вольт-амперные характеристики эмиттеров с остриями высотой $h=30 \mu \mathrm{m}$ и с радиусом закругления вершины $R=20 \mathrm{~nm}$ для структур с разными значениями расстояния $L$ между остриями. Площадь эмиттера $S=0.2 \mathrm{~cm}^{2}$. Вольт-амперные характеристики рассчитаны до максимального анодного тока, при котором ток с одиночного острия достигает значения $5 \mu \mathrm{A}$.

теристик для многоострийных эмиттеров с остриями высотой $h=30 \mu \mathrm{m}$ и с радиусом вершины $R=20 \mathrm{~nm}$, полученных для разных значений расстояния $L$ между остриями. Расчет велся до достижения предельного тока $5 \mu \mathrm{A} \mathrm{c} \mathrm{острия.} \mathrm{Из} \mathrm{полученных} \mathrm{данных} \mathrm{следует,} \mathrm{что} \mathrm{в}$ условиях, когда практически отсутствует экранировка (при $L=60 \mu \mathrm{m}=2 h$ ), эмиттер площадью $0.2 \mathrm{~cm}^{2}$ может обеспечить токи не более $25 \mathrm{~mA}$. Большие токи могут быть получены с эмиттера той же площади, но с большим количеством острий на поверхности и при большем напряжении между катодом и анодом. Определяет выбор соотношения высоты острий и расстояния между ними допустимое значение напряжения $U$ при эксплуатации катода. Проведенные расчеты свидетельствуют (рис. 6), что, например, при $U=17 \mathrm{kV}$ многоострийная структура острий высотой $30 \mu \mathrm{m}$ при $L / h=0.5$ может обеспечить токи полевой эмиссии $\sim 0.4 \mathrm{~A}$.

\section{Заключение}

Подытоживая результаты выполненной работы, отметим важнейшее:

- в численных расчетах получены данные о влиянии на эмиссионные характеристики острийных кремниевых полевых эмиттеров с двуслойными металл-фуллереновыми покрытиями высоты и радиуса закругления вершины острий, а также расстояния между остриями, необходимые для оптимизации морфологии их поверхности;

- уточнены закономерности взаимной экранировки острий в многоострийных системах. Полученные данные свидетельствуют о целесообразности эксплуатации многоострийных полевых эмиттеров в высоковольтных 
электронных приборах в условиях частичной взаимной экранировки;

- продемонстрирована возможность получения с многоострийного кремниевого эмиттера площадью $0.2 \mathrm{~cm}^{2}$ с двухслойным металл-фуллереновым покрытием токов эмиссии до нескольких сотен миллиампер.

Исследование выполнено за счет гранта Российского научного фонда (проект № 16-12-10010).

\section{Список литературы}

[1] Glyavin M.Yu., Chirkov A.V., Denisov G.G. et al. // Rev. Sci. Instrum. 2015. Vol. 86. P. 054705.

[2] Booske J.H., Dobbs R.J., Joye C.D., Kory C. L., Neil G.R., Park G.-S., Park J., Temkin R.J. // IEEE Trans. Terahertz Sci. Technol. 2011. Vol. 1. N 1. P. 54-75.

[3] Wang S., Calderon X., Peng R. et al. // Appl. Phys. Lett. 2011. Vol. 98. P. 213701.

[4] Ooki S., Ohsio S., Nishino J. et al. // Jap. J. Appl. Phys. 2008. Vol. 47. P. 7303.

[5] Соминский Г.Г., Сезонов В.Е., Тарадаев Е.П., Тумарева Т.А., Задиранов Ю.М., Корнишин С.Ю., Степанова А.Н. // Изв. вузов. Радиофизика. 2015. Т. 58. Вып. 7. C. $567-576$.

[6] Соминский Г.Г., Тарадаев Е.П., Тумарева Т.А., Гиваргизов М.Е., Степанова А.Н. // ЖТФ. 2016. Т. 86. Вып. 11. C. $108-111$.

[7] Тумарева Т.А., Соминский Г.Г., Светлов И.А., Морозов А.Н. // ЖТФ. 2008. Т. 78. Вып. 11. С. 119-122.

[8] Тумарева Т.А., Соминский Г.Г. // Изв. вузов. Прикладная нелинейная динамика. 2009. Т. 17. Вып. 3. С. 17-54.

[9] Тумарева Т.А., Соминский Г.Г. // ЖТФ. 2013. Т. 83. Вып. 7. C. $121-124$.

[10] Соминский Г.Г., Тумарева Т.А. // Изв. вузов. Прикладная нелинейная динамика. 2015. Т. 23. Вып. 2. С. 74-93.

[11] Соминский Г.Г., Тумарева Т.А., Тарадаев Е.П., Мишин М.В., Степанова А.Н. // ЖТФ. 2015. Т. 85. Вып. 1. C. $138-141$.

[12] Гиваргизов Е.И. // Кристаллография. 2006. Т. 51. Вып. 5. C. 947-953.

[13] Пат. РФ № 151235. Острийный полевой эмиттер/ Г.Г. Соминский, Е.П. Тарадаев, Т.А. Тумарева. 2015. БИ N 9.

[14] Бочаров Г.С., Елечкий А.В. // ЖТФ. 2005. Т. 75. Вып. 7. C. 126.

[15] Бочаров Г.С., Елецкий А.В., Sommerer T.J. // ЖТФ. 2011. Т. 81. Вып. 4. С. 111-116. 